\title{
A Review on Decongesting Urban Traffic With Smart Mobility
}

\author{
Rupinder Pal Singh \\ Ramgarhia Institute of engg. \\ \& Technology Phagwara
}

\author{
Dr. Naveen Dhillon \\ Principal at Ramgarhia \\ Institute of engg. \& \\ Technology Phagwara
}

\author{
Prof.Manit Kapoor \\ HOD of ECE department at \\ Ramgarhia Institute of engg. \\ \& Technology Phagwara
}

\begin{abstract}
The traffic chaos is getting more and more serious problem across the urban areas of the nation, which is causing the hours long traffic jams. The traffic jams cause the more fuel consumption, high carbon emissions, long wait for office or other places for people and several other problems, which are directly or indirectly causing the problem in public life and environment. Addressing both of the factors remains necessary to increase the quality of life in the means of commute times and clean air. In this paper, the problem of congestion has been addressed by using applying the contention management application under the proposed model, which controls the flow of the vehicles in multiple directions on the basis of vehicular density. The windowing mechanism is coupled with the contention management, which dynamically controls the vehicular movement from each direction. This paper evaluates the vehicles moving on the different speeds with the congestion (no. of vehicles), pollution level and consumption of fuel based parameters.
\end{abstract}

KEYWORDS - Smart Contention management, Contention window control, Vehicular Network, Traffic Management

\section{INTRODUCTION}

VANET stands for Vehicular Ad hoc Network. VANET, is a technology in which cars act as a nodes to make a mobile network. [1] A VANET each car is act like a wireless router or node, with range of transmission data of one hundred to three hundred meters produce a wide network. [2] Due to movement of cars, different cars will take part in network, and form a network by connecting with at least one vehicle [3].

In vanet main problem is congestion. So Vehicular networks are such designed that will give us better security, and remove congestion of the transportation systems [5]. To remove the congestion it is better to run intelligent road traffic management system. In Future intelligent road traffic management systems are expected to supply many congestion controls is used to present better traffic management systems. [6] To send congestion information RSU (Road side unit) is used. This unit takes all the information like hazard, car break etc from the cars and send to the different cars.

\section{PROBLEM FORMULATION}

The existing model is entirely based upon the cooperative VANETs and facilitates to exchange the positioning and status information between VANET neighbors. The exchange of messages up to 1-hop is considered as the control channels in periodic fashion. The dynamic network adaptation ability of the automated vehicular networks on the basis of transmission parameters require the efficient approaches to effectively handle the congestion 
by the effective congestion control protocols to control the channel load, typically through the adaptation of the transmission parameters based on certain channel load metrics. To date, congestion and awareness management protocols are ordinarily designed and evaluated severally, though each are needed for the reliable and economical operation of conveyance networks. During this context, this paper proposes the devaluate INTERN solution, a replacement management protocol that integrates two congestion and awareness management processes. The simulation results obtained for 3 completely different eventualities demonstrate that INTERN is in a position to satisfy the applications' needs of all vehicles, whereas effectively dominant the channel load. The results obtained highlight the challenges ahead with rising machine-controlled vehicles.

The existing model hasn't been tested with the highly dense traffic congestion where most of the vehicular models fail. The highly congested periods in the VANETs must be tackled with more intelligence and highly planned traffic shaping \& route planning. The existing model does not incorporate any of the quality of service (QoS) approach to segregate the traffic according to its important and also does not cluster the destination nodes for whom the aimed traffic classified as super critical. The existing model does not utilize the load balancing approach to minimize the traffic load over single link. The load balancing approaches can help out the failover management. Also the load balancing approach minimizes the dependency of the intermediate nodes across one path. The existing model does not utilize any message compression or optimization method. The optimal message compression or optimization methods are considered effective in reducing the overall load from the VANET link by reducing the traffic size. The optimization techniques are considered better than compression because there is no need to decompress them.

\section{METHODOLOGY}

This research project will start with a detailed literature review on the various VANET mobility and collision avoidance and coverage schemes. Then, a detailed coverage and connectivity mechanism would be designed to prevent the issue of non-connected nodes and to provide the maximum message reach in VANETs. The simulation would be implemented using Network Simulator (NS2). The obtained results would be examined and compared with the existing security mechanism to address the similar issues. Waterfall development method is ideal for projects with clear task formalization and fixed scope of work like this research work, i.e. for small and medium-size projects.

\section{CONCLUSION}

The vehicular network is the solution to connect the multiple vehicles across the cities to the nationwide vehicular network, which allows the solution designers to apply the collected information for various applications. One most popular application of the vehicular networks is to evaluate the congestion across the different points of cities, and to manage the signal resources effectively to clear the congestion. The dynamic and dependent control of the light points on the basis of different density parameters evaluated from the location of the vehicles collected through the vehicular network. The traffic decongestion improves the multiple factors of the urban traffic, which eventually improvise the quality of environment by reducing the pollutant (specifically carbon and particulate matter, PM2..5 \& PM10) emissions. Also it improves the quality of life by reducing the commuters time to office and back to home, which gives them more daily time for their personal activities, and eventually increases the quality of life of residents. 


\section{FUTURE SCOPE}

In the future, we will implement the proposed model with different scenarios in order to evaluate its performance, the results of which will be evaluated against the previous studies in order to understand the improvement factor.

\section{REFERENCES}

I. Abdalla, Ghassan MT, Mosa Ali Abu-Rgheff, and Sidi Mohammed Senouci. "Current trends in vehicular ad hoc networks." Proceedings of UBIROADS workshop. 2007.

II. Bibhu, Vimal, et al. "Performance Analysis of black hole attack in VANET." International Journal of Computer Network and Information Security (IJCNIS) 4.11 (2012): 47.

III. Chen, Lu, Hongbo Tang, and Junfei Wang. "Analysis of VANET security based on routing protocol information." Intelligent Control and Information Processing (ICICIP), 2013 Fourth International Conference on. IEEE, 2013.

IV. Chim, Tat Wing, et al. "SPECS: Secure and privacy enhancing communications schemes for VANETs." Ad Hoc Networks 9.2 (2011): 189-203.

V. Chim, Tat Wing, et al. "Security and privacy issues for inter-vehicle communications in VANETs." Sensor, Mesh and Ad Hoc Communications and Networks Workshops, 2009. SECON Workshops' 09. 6th Annual IEEE Communications Society Conference on. IEEE, 2009. Dias, João A., et al. "Testbed-based performance evaluation of routing protocols for vehicular delay-tolerant networks."GLOBECOM Workshops (GC Wkshps), 2011 IEEE. IEEE, 2011.

VI. Douceur, John R. "The sybil attack." Peer-to-peer Systems. Springer Berlin Heidelberg, 2002. 251-260.

VII. Ghaleb, Fuad A., M. A. Razzaque, and Ismail FauziIsnin. "Security and privacy enhancement in VANETs using mobility pattern." Ubiquitous and Future Networks (ICUFN), 2013 Fifth International Conference on. IEEE, 2013.

VIII. Guette, Gilles, and Ciarán Bryce. "Using TPMs to secure vehicular ad-hoc networks (VANETs)." Information Security Theory and Practices. Smart Devices, Convergence and Next Generation Networks. Springer Berlin Heidelberg, 2008. 106116

IX. Guette, Gilles, and Bertrand Ducourthial. "On the Sybil attack detection in VANET." Mobile Adhoc and Sensor Systems, 2007. MASS 2007. IEEE Internatonal Conference on. IEEE, 2007.

X. Hu, Yih-Chun, Adrian Perrig, and David B. Johnson. "Packet leashes: a defense against wormhole attacks in wireless networks." INFOCOM 2003. Twenty-Second Annual Joint Conference of the IEEE Computer and Communications. IEEE Societies. Vol. 3. IEEE, 2003.

XI. Hung, Chia-Chen, Hope Chan, and EH-K. Wu. "Mobility pattern aware routing for heterogeneous vehicular networks." Wireless Communications and Networking Conference, 2008. WCNC 2008. IEEE. IEEE, 2008.

XII. Javed, Muhammad A., and Jamil Y. Khan. "A Geocasting technique in an IEEE802. 11p based vehicular ad hoc network for road traffic management." Australasian Telecommunication Networks and Applications Conference (ATNAC), 2011. IEEE, 2011.

XIII. Khabazian, Mehdi, and M. K. Mehmet Ali. "Generalized performance modeling of vehicular Ad Hoc networks (VANETs)." Computers and Communications, 2007. ISCC 2007. 12th IEEE Symposium on. IEEE, 2007. 
XIV. Leinmüller, Tim, et al. "Improved security in geographic ad hoc routing through autonomous position verification." Proceedings of the 3rd international workshop on Vehicular ad hoc networks. ACM, 2006.

XV. Lo, Nai-Wei, and Hsiao-Chien Tsai. "Illusion attack on VANET applicationsA message plausibility problem." Globecom Workshops, 2007 IEEE. IEEE, 2007.

XVI. Moser, Steffen, Simon Eckert, and Frank Slomka. "An approach for the integration of smart antennas in the design and simulation of vehicular ad-hoc networks." Future Generation Communication Technology (FGCT), 2012 International Conference on. IEEE, 2012.

XVII. Mahmood, Raja, and A. I. Khan. "A survey on detecting black hole attack in AODV-based mobile ad hoc networks." High Capacity Optical Networks and Enabling Technologies, 2007. HONET 2007. International Symposium on. IEEE, 2007.

XVIII. Mahmood, Raja, and A. I. Khan. "A survey on detecting black hole attack in AODV-based mobile ad hoc networks." High Capacity Optical Networks and Enabling Technologies, 2007. HONET 2007. International Symposium on. IEEE, 2007.

XIX. Malla, AdilMudasir, and Ravi Kant Sahu. "Security Attacks with an Effective Solution for $\mathrm{S}$ Attacks in VANET."International Journal of Computer Applications 66.22 (2013).

XX. Manvi, S. S., M. S. Kakkasageri, and D. G. Adiga. "Message authentication in vehicular ad hoc networks: Ecdsa based approach." Future Computer and Communication, 2009. ICFCC 2009. International Conference on. IEEE, 2009.

XXI. M. Khabazian, M. K. Mehmet Ali, "A Performance Modeling of Vehicular Ad Hoc Networks (VANETs)", 2007 IEEE.

XXII. Park, Soyoung, et al. "Defense against sybil attack in vehicular ad hoc network based on roadside unit support."Military Communications Conference, 2009. MILCOM 2009. IEEE. IEEE, 2009.

XXIII. Parno, Bryan, and Adrian Perrig. "Challenges in securing vehicular networks." Workshop on hot topics in networks (HotNets-IV). 2005.

XXIV. Paxson, Vern. "Bro: a system for detecting network intruders in realtime." Computer networks 31.23 (1999): 2435-2463.

XXV. Qian, Yi, Kejie Lu, and Nader Moayeri. "Performance evaluation of a secure MAC protocol for vehicular networks." Military Communications Conference, 2008. MILCOM 2008. IEEE. IEEE, 2008. 